\title{
FROM THE EDITOR
}

\section{Thematic issue "Heterocycles against viruses"}

In recent decades, viral infections have become a serious threat to the well-being and security of humans, society, and civilization. The mortality rate of new viral diseases turned out to be exorbitantly high in comparison with traditional epidemics. The development of such diseases is caused by many factors of the globalization of civilizational processes, and mankind will have to respond to these challenges. The current rapid and protracted COVID-19 pandemic has shown the lack of attention of the world scientific community to conditionally safe viral infections.

Humanity is not insured from the relapse of such a pandemic scenario until reliable and universal approaches to the creation of effective antiviral agents, including compounds with broad spectrum of action, would be developed, since the use of medicinal compounds, as small molecules, is the most important way to combat viral diseases.

The focus of this thematic issue of the journal is on the heterocyclic compounds as molecules that occupy a special place among antiviral agents. For medicinal chemistry, as for any other rapidly developing field of knowledge, it is advisable to systematize and analyze the accumulated experience. Therefore, the thematic issue includes 8 review articles on various classes of heterocyclic compounds that have a high potential for medical use as antiviral drugs. The arsenal of tools, methods, and theoretical approaches to medicinal chemistry in recent years has been significantly enriched, expanded and a significant role is now played by predictive calculations of the possibility of small molecules, including heterocycles, for interaction with biological macrostructures responsible for the development of infectious processes, which is reflected in this issue of the journal. At the same time, synthetic organic chemistry, as before, plays a decisive role in the successful creation of new drug candidates. In this issue, 12 experimental works on the targeted synthesis of new derivatives of diverse types of heterocycles with variable antiviral activity are published.

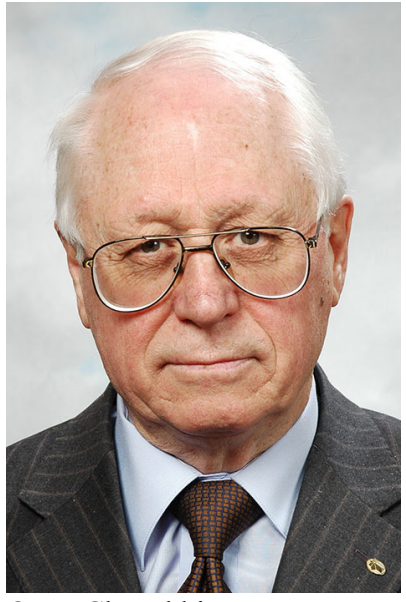

O. N. Chupakhin

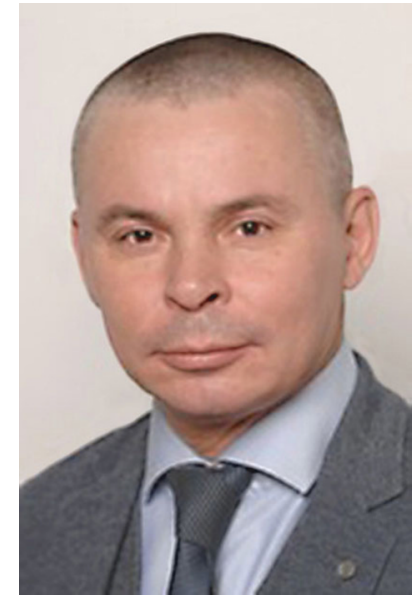

E. N. Ulomsky
Let us personally and on behalf of the editorial board of the journal "Chemistry of Heterocyclic Compounds" thank all the colleagues who contributed to this thematic issue. We would like to express our special gratitude to the reviewers, whose objective criticism and comments helped to improve the quality of the submitted works, and we would also like to thank the staff of the Editorial Office of the journal who participated at all stages of work.

We hope that the result of our joint work will be interesting to all specialists working in the field of chemistry of biologically active heterocyclic compounds and will contribute to the creation of modern drugs.

Guest Editors of the thematic issue

O. N. Chupakhin, academician of the RAS, E. N. Ulomsky, PhD, Professor, Ural Federal University named after the first President of Russia B. N. Yeltsin, Postovsky Institute of Organic Synthesis, Ural Branch of the Russian Academy of Sciences 